\title{
Convulsive and Neurodegenerative Effects in Rats of Some Isolated Toxins from the Tityus bahiensis Scorpion Venom
}

\author{
Luciene Toshie Takeishi Ossanai, ${ }^{1,2}$ Geane Antiques Lourenço, ${ }^{1}$ Ivo Lebrun, ${ }^{3}$ \\ Ana Leonor Abrahão Nencioni, ${ }^{1}$ and Valquiria Abrão Coronado Dorce ${ }^{1}$ \\ ${ }^{1}$ Laboratory of Pharmacology, Butantan Institute, Avenida Dr. Vital Brazil 1500, 05503-900 São Paulo, SP, Brazil \\ ${ }^{2}$ Disease Control Coordinating Agency of São Paulo State Public Health Ministry, 01246-000 São Paulo, SP, Brazil \\ ${ }^{3}$ Laboratory of Biochemistry and Biophysics, Butantan Institute, 05503-900 São Paulo, SP, Brazil \\ Correspondence should be addressed to Valquiria Abrão Coronado Dorce; vdorce@butantan.gov.br
}

Received 27 June 2013; Revised 10 September 2013; Accepted 13 September 2013

Academic Editor: Andreimar Martins Soares

Copyright (C) 2013 Luciene Toshie Takeishi Ossanai et al. This is an open access article distributed under the Creative Commons Attribution License, which permits unrestricted use, distribution, and reproduction in any medium, provided the original work is properly cited.

\begin{abstract}
Despite Tityus bahiensis being one of the most dangerous scorpions in Brazil, there are few studies about the effects of its venom, which acts mainly on the central nervous system. Previous studies demonstrated the convulsive ability of this venom. The present work aimed to study the hippocampal effects in rats of some toxins isolated from pool $\mathrm{V}$, which induces a pronounced epileptogenic effect. The pool was separated by reverse-phase HPLC, and the peaks with higher yield (Tb V-1, V-5, V-24, V-27, and V-28) were used in the experiments. Cannulae and electrodes were implanted in the hippocampus of male Wistar rats $(240-250 \mathrm{~g})$. The animals were divided into six groups that received intracerebral injection of toxin solution ( 1 or $2 \mu \mathrm{g} / \mu \mathrm{L}$ ) or Ringer solution (control group), and they were submitted to behavioral, electrographic, and histological analysis. All toxins studied evoked electrographic and behavioral epileptic-like activity to different degrees. Moreover, the toxins V-1, V-24, and V-28 caused significant neuronal loss in CA4 ipsi- and contralateral hippocampal areas. These results suggest that toxins from T. bahiensis scorpion, when injected into the hippocampus, are able to act directly on the central nervous system inducing convulsive and neurodegenerative effects.
\end{abstract}

\section{Introduction}

From an epidemiological viewpoint, the scorpion family Buthidae is considered to be the most important among the 19 families distributed in the world [1]. Twenty-five species dangerous to humans belong to this family. In Brazil, scorpions from the genus Tityus, which belong to this family, represent about $60 \%$ of the neotropical fauna [2], and the most important species are Tityus serrulatus, $T$. bahiensis, $T$. stigmurus, and T. obscurus [3].

The purification and sequencing of toxin from T. bahiensis were initially done by Becerril et al. [4], who compared them with toxins from $T$. serrulatus and observed that the most abundant peptide from this scorpion (named $\gamma$ bahiensis) is $95 \%$ identical to toxin $\gamma$ from the venom of Tityus serrulatus. Other toxins have already been isolated, such as III- 8 bahiensis (III- $8 \mathrm{~b}$ ), TbTx-VI, and IV-5 bahiensis
(IV-5b), which show $80 \%$ homology with toxin IV-5 from $T$. serrulatus [5].

Meanwhile, there are few papers focusing on the purification, sequencing, mechanisms of action, and effects of toxins that comprise this venom [4-7].

In spite of the large number of studies in the literature as revised by Cologna et al. [3], the central effects of toxins and venoms have been neglected due to the common understanding that these peptides do not cross the bloodbrain barrier [8-10]. However, Clot-Faybesse et al. [11] found labeled toxins in the central nervous system after subcutaneous injection in newborn mice, in which the barrier is not totally developed. Additionally, Nunan et al. [12] and Guidine et al. [13] observed the presence of TsTX toxin in the brain of young rats after subcutaneous injection, indicating that the toxin crosses the brain barrier. Moreover, Nencioni et al. [14] demonstrated central changes in levels of homovanillic 
acid, a metabolite of the dopamine neurotransmitter, after intraperitoneal injection of venom of both T. serrulatus and T. bahiensis.

Lourenço et al. [6] demonstrated that the venom from T. bahiensis scorpion induces convulsions and that fractions (nonpurified toxins) from this venom, when injected intravenously, evoke alterations in the central nervous system, reinforcing the idea that toxins are able to cross the bloodbrain barrier.

All these results suggest the importance of toxins in the central nervous system in cases of severe envenomation, which may explain the occurrence of more serious accidents in patients in which the blood-brain barrier is weak, such as in the elderly, or not totally developed, such as in children [10].

On the basis of available studies and on the data of Lourenço et al. [6], the present study aimed to determine the effects of some toxins isolated from T. bahiensis scorpion venom on the central nervous system of rats when injected into the hippocampus.

\section{Materials and Methods}

2.1. Subjects. Male Wistar rats (240-250 g) were used. Upon their arrival in the laboratory (seven days before the experiments), the animals were individually housed in wire mesh cages and kept at constant temperature $\left(22 \pm 1^{\circ} \mathrm{C}\right)$, in a 12hour light/12-hour dark cycle (lights on at $0700 \mathrm{~h}$ ), with food and water provided ad libitum. All experimental procedures were approved by our Institutional Ethics Committee for Experiments on Animals (462/08).

2.2. Purification of Toxins. Fractions (or pools) were isolated from $50 \mathrm{mg}$ venom diluted in $500 \mu \mathrm{L}$ of $50 \mathrm{mM}$ ammonium bicarbonate buffer. The solution was homogenized and centrifuged $(5,000 \mathrm{rpm})$, and the supernatant was stored in the refrigerator. The precipitate was resuspended with $500 \mu \mathrm{L}$ of the same buffer, which was then homogenized and centrifuged. The resulting supernatant was added to the first one, and the final total volume obtained was $1.0 \mathrm{~mL}$. The final supernatant was applied to a Sephadex G50 column $(0.5 \mathrm{~cm}$ diameter and $78 \mathrm{~cm}$ length), with a total volume of $245 \mathrm{~mL}$ and $49 \mathrm{~mL}$ of dead volume, which was discarded. Fractions were obtained and pooled using a Bio-Rad automated fraction collector. Chromatography was performed with a flow rate of $9.0 \mathrm{~mL} / \mathrm{h}$. The eluate was monitored with a spectrophotometer at $280 \mathrm{~nm}$ and $1.5 \mathrm{~mL}$ fractions were collected. The pooled fractions were then lyophilized and stored at $-20^{\circ} \mathrm{C}$ until use.

For the purification of toxins, the pools of interest were resuspended in $1.5 \mathrm{~mL}$ of ultrapure water and separated by high-performance liquid chromatography (HPLC-HP 1100 series) using a C18 analytical column (Beckman Ultrasphere $5 \mu \mathrm{m}, 250 \mathrm{~mm} \times 4.6 \mathrm{~mm}$ ) with detection in a spectrophotometer (HP) at a wavelength of $214 \mathrm{~nm}$. Elution was carried out using $0.1 \%$ trifluoroacetic acid in ultrapure water (solvent A) as the polar phase and $90 \%$ acetonitrile with $10 \%$ solvent A (solvent B) as the apolar phase. The material applied to the
HPLC was subjected to a 30-minute run with a linear gradient of $10-70 \%$ solvent $\mathrm{B}$.

Peaks were manually collected from the starting point to the end without any overlap with other peaks in order to reach at least 95\% of purity, HPLC grade, except for peaks 27 and 28 that need further purification. The amount of material in each peak was estimated by the area under the peak. The different peaks of material were collected, freeze-dried, and stored at $-20^{\circ} \mathrm{C}$ until use.

2.3. Surgery. The animals were anesthetized $(3 \mathrm{~mL} / \mathrm{kg})$ with a mixture of pentobarbitone $(1.0 \mathrm{~g})$ and chloral hydrate $(4.0 \mathrm{~g})$ in $100 \mathrm{~mL}$ of $0.9 \% \mathrm{NaCl}$ and submitted to stereotaxic surgery. Stainless steel guide cannulae were chronically implanted in the dorsal hippocampus for intracerebral injection, and bipolar twisted Teflon-coated stainless-steel electrodes were positioned on the opposite side of the dorsal hippocampus for depth recordings. Coordinates were derived from a stereotaxic atlas (AP -4.8, L 3.2, V 2.8 for cannula and AP -4.8 , L 3.2, V 3.0 for electrodes) [15]. Surface recordings were obtained with jeweler screws positioned bilaterally over the occipital cortex. An additional screw placed in the frontal sinus served as reference (indifferent electrode). After surgery, animals were housed individually and were allowed to recover for a period of 1-2 days.

\subsection{Electroencephalographic Recordings and Behavioral} Observations. Electroencephalographic recordings (EEG) and behavioral observations were carried out in a glass compartment placed in a Faraday cage. The bipolar twisted electrodes of each animal were connected to a PowerLab recording apparatus (AD Instruments, Canada), and after 15 minutes of acclimation to the test cage, the baseline electroencephalographic trace of the cortex and hippocampus was recorded for $15 \mathrm{~min}$. The electrodes were then disconnected and the animals were submitted to the intracerebral injection. Seventy-three rats were submitted to the surgery; however, only rats with correctly implanted electrodes and cannulae were used in this study. The rats were divided into 6 groups and submitted to intrahippocampal injection of (i) Ringer solution administered in an injection volume of $1 \mu \mathrm{L}(n=8)$, (ii) Tb V-1 at a dose of $1 \mu \mathrm{g} / \mu \mathrm{L}$ ( $n=12)$, (iii) $\mathrm{Tb} \mathrm{V}-5$ at $1 \mu \mathrm{g} / \mu \mathrm{L}(n=13)$, (iv) $\mathrm{Tb} \mathrm{V}-24$ at $2 \mu \mathrm{g} / \mu \mathrm{L}(n=8),(\mathrm{v}) \mathrm{Tb} \mathrm{V}-27$ at $2 \mu \mathrm{g} / \mu \mathrm{L}(n=8)$, and (vi) $\mathrm{Tb} \mathrm{V}-28$ at $2 \mu \mathrm{g} / \mu \mathrm{L}$ of $(n=8)$. An intracerebral injection at a rate of $1.0 \mu \mathrm{L} / \mathrm{min}$ was performed with a $5 \mu \mathrm{L}$ Hamilton microsyringe connected through a polyethylene tubing to a dental needle. The needle was kept for 30 seconds into the guide cannula after the end of the injection in order to avoid reflow. The electrodes were immediately reconnected. For all toxins, the volume of $1.0 \mu \mathrm{L}$ was used. All toxins were previously tested. The toxins that caused behavioral and/or electrographic alterations were chosen to perform the experiments. The doses were determined in these experiments.

Subsequently, continuous EEG recording and observations of animal behavior were performed for a period of 
4 h. Signals were amplified using EEG100C amplifiers (highpass filter, $1 \mathrm{~Hz}$; low-pass filter, $100 \mathrm{~Hz}$; 5,000_gain), digitized at $500 \mathrm{~Hz}$ using an MP150 digital-analog converter, and acquired with AcqKnowledge acquisition software (BioPac Systems, Santa Barbara, CA). The electrographic parameters assessed were isolated or grouped spikes and short, moderate, or strong epileptic-like discharges. The behavioral parameters were yawning, orofacial automatism, increased secretions and salivation, myoclonus, and "wet dog shakes" (WDS).

Fisher's test was used to analyze the behavioral parameters and electrographic alterations $(P<0.05)$.

2.5. Histology. Neuronal integrity and the correct location of implanted deep electrodes and cannulae were checked histologically. The morphological analysis was performed 7 days after toxin injection. Seven days is the time required to verify whether neurodegeneration occurs. The animals were deeply anesthetized with $\mathrm{CO}_{2}$ and perfused through the heart (left ventricle) with phosphate-buffered saline (PBS) followed by $10 \%$ formalin solution. The brains were removed, stored in formalin, and embedded in Paraplast. Coronal brain sections of $10 \mu \mathrm{m}$ were cut from a $700-\mu \mathrm{m}$ brain block including the cannula track. The slices were mounted on a glass slide and stained with cresyl violet. The number of intact cells in the CA1, CA3, and CA4 hippocampal areas was determined by light microscopy using a 40x objective. A two-dimensional cell count was performed using a $100 \times 100 \mu \mathrm{m}$ grid. Only pyramidal neurons located inside the reticulum area, with a visible nucleus and nucleolus, were considered intact.

ANOVA followed by Tukey's test was used for statistical analysis $(P<0.05)$.

\section{Results}

3.1. Purification of Toxins. Fifty milligrams of crude $T$. bahiensis venom was extracted twice with ammonium bicarbonate buffer and centrifuged at 5,000 rpm. The supernatant was applied to a Sephadex G-50 M column and the precipitate was discarded. Six pools were obtained according to OD values (Figure 1(a)).

Due to the similar effects between pool V, tested in the present study, and pool VI, previously obtained by Lourenço et al. [6], which had a pronounced epileptogenic effect, pool $\mathrm{V}$ was chosen and separated by HPLC, resulting in 30 peaks (Figure 1(b)). Some isolated toxins were chosen according to their effect and yield, namely, Tb V-1, Tb V-5, Tb V-24, Tb $\mathrm{V}-27$, and $\mathrm{Tb} \mathrm{V}-28$.

3.2. Electroencephalographic Recordings and Behavioral Observations. Animals injected with $\mathrm{Tb} \mathrm{V}-1$ toxin displayed behavioral alterations such as yawning, orofacial automatisms, myoclonus, WDS, and increased salivary and/or nasal secretions (Table 1). The electrographic changes (Figure 2) started approximately 2 minutes after toxin injection and were characterized by isolated and grouped spikes and short discharges (Table 2).

Tb V-5 caused yawning, orofacial automatisms, myoclonus, and WDS (Table 1). The electrographic alterations
(Figure 2) started 6 minutes after the injection and were characterized by isolated and grouped spikes and short discharges (Table 2).

$\mathrm{Tb}$ V-24 was responsible for yawning, orofacial automatisms, myoclonus, WDS, and increased salivary and/or nasal secretions (Table 1). This toxin showed the shortest time to evoke electrographic alterations (1 minute), such as isolated or grouped spikes and short and moderate discharges (Table 2).

$\mathrm{Tb}$ V-27 enhanced yawning, orofacial automatisms, myoclonus, WDS, and increased salivary and/or nasal secretions (Table 1). Electrographic alterations (Figure 2) occurred approximately 5 minutes after the injection. The alterations observed were isolated and grouped spikes and short, medium, and strong discharges (Table 2).

Behavioral alterations caused by $\mathrm{Tb} \mathrm{V}-28$ toxin were yawning, orofacial automatisms, myoclonus, and WDS (Table 1). Electrographic alterations (Figure 2) started approximately 5 minutes after the injection and were characterized by isolated and grouped spikes and short, medium, and strong discharge (Table 2).

Intracerebral injection of Ringer solution did not alter behavior or electroencephalographic recordings (Tables 1 and 2).

3.3. Histology. A significant decrease was seen in the number of cells in CA4 ipsi- and contralateral areas after the injection of TbV-1, Tb V-24, and Tb V-28 toxins. The number of cells was not significantly altered in the other areas, although a slight decrease was observed in some histological slices (Figures 3 and 4).

In animals injected with $\mathrm{Tb} \mathrm{V}-5, \mathrm{~Tb} \mathrm{~V}-27$, and Ringer solution, there was no change in the number of cells in all the areas analyzed (Figures 3 and 4).

\section{Discussion}

Toxins isolated from the scorpion venoms can be useful as neurological tools for studying convulsive activities and the function of ionic channels.

It was previously found that T. bahiensis scorpion venom causes a more intense convulsion than that evoked by $T$. serrulatus [6]. The initial screening of pools purified from this venom demonstrated that pool $\mathrm{V}$ has a more acute epileptogenic effect. Thus, in the present study, this pool was further purified to obtain isolated toxins, and some of them, according to their yield, were chosen to be tested.

The hippocampus was determined as the site of study because it is involved in the epileptogenic process and is utilized in several models of epilepsy [16]. It is also easily accessible and recognizable in histological analysis.

It is known that the venoms of scorpions from the family Buthidae have a large quantity of toxins that modulate sodium channel function [17], stimulating neurotransmitter release from cholinergic, adrenergic, glutamatergic, and GABAergic systems in synaptosomes of rats [18-20] and in the hippocampus in vivo [21]. 


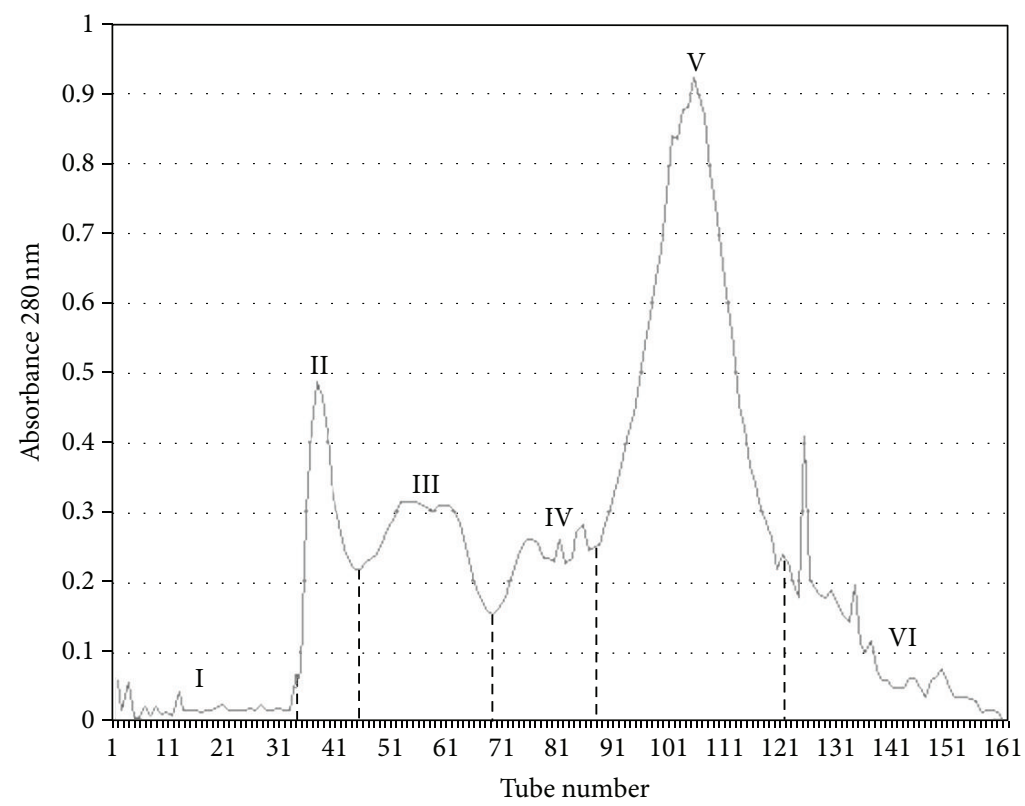

(a)

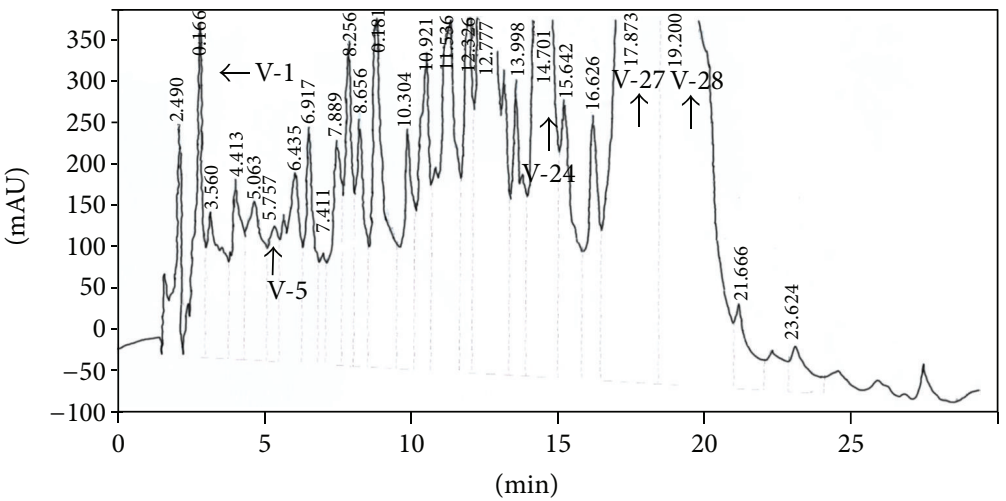

(b)

Figure 1: (a) Sephadex G-50 M chromatography of the Tityus bahiensis scorpion venom after extraction with $0.05 \mathrm{M}$ ammonium bicarbonate buffer, $\mathrm{pH}$ 8.0, eluted with the same buffer. The eluate was monitored at $280 \mathrm{~nm}$, and $1.5-\mathrm{mL}$ fractions were collected. (b) Analytical HPLC profile of the G-50 active peak on a C18 analytical column eluted with trifluoroacetic acid (0.1\%) (solvent) and acetonitrile (90\%) (solvent) and a solvent gradient developed from 10-70\% within 30 minutes and monitored at $214 \mathrm{~nm}$. Arrows indicate the used toxins.

TABLE 1: Behavioral effects of injection of Tityus bahiensis scorpion venom toxins into the hippocampus. The rats had cannulae and electrodes implanted in the CA1 area of the hippocampus. The observations were made up to $4 \mathrm{~h}$ after injection.

\begin{tabular}{|c|c|c|c|c|c|}
\hline & Yawning & $\begin{array}{c}\text { Orofacial } \\
\text { automatisms }\end{array}$ & $\begin{array}{l}\text { Increased salivary and/or nasal } \\
\text { secretions }\end{array}$ & Myoclonus & Wet dog shakes (WDS) \\
\hline Ringer $(n=8)$ & - & - & - & - & - \\
\hline $\mathrm{Tb} \mathrm{V}-1(n=12)$ & $83.33 \%^{*}$ & $83.33 \%^{*}$ & $41.6 \%$ & $100 \%^{*}$ & $83.33 \%^{*}$ \\
\hline $\mathrm{Tb} \mathrm{V}-5(n=13)$ & $92.31 \%^{*}$ & $76.92 \%^{*}$ & - & $84.46 \%{ }^{*}$ & $61.54 \%{ }^{*}$ \\
\hline $\mathrm{Tb} \mathrm{V}-24(n=8)$ & $50 \%{ }^{*}$ & $33.33 \%$ & $16.6 \%$ & $83.33 \%^{*}$ & $50 \%{ }^{*}$ \\
\hline $\mathrm{Tb} \mathrm{V}-27(n=8)$ & $25 \%$ & $37.5 \%$ & $37.5 \%$ & $37.5 \%$ & $50 \%^{*}$ \\
\hline Tb V-28 $(n=8)$ & $12.5 \%$ & $62.5 \%{ }^{*}$ & - & $75 \%{ }^{*}$ & $62.5 \%{ }^{*}$ \\
\hline
\end{tabular}

*Significantly different from control group treated with Ringer solution; $P<0.05$, Fisher's exact test. 
TABLE 2: Electrographic effects of injection of Tityus bahiensis scorpion venom toxins into the hippocampus. The rats had cannulae and electrodes implanted in the CA1 area of the hippocampus. The recordings were made up to $4 \mathrm{~h}$ after injection. The group of animals that presented a significant increase in the electrographic signs was considered as having epileptiform activity.

\begin{tabular}{|c|c|c|c|c|c|}
\hline & Isolated spikes & Grouped spikes & Short discharge & Moderate discharge & Strong discharge \\
\hline Ringer & - & - & - & - & - \\
\hline Tb V-1 & $100 \%^{*}$ & $100 \%^{*}$ & $75 \%^{*}$ & - & - \\
\hline Tb V-5 & $100 \%{ }^{*}$ & $50 \%{ }^{*}$ & $100 \%^{*}$ & - & - \\
\hline $\mathrm{Tb} \mathrm{V}-24$ & $100 \% *$ & $100 \% *$ & $40 \%$ & $25 \%$ & - \\
\hline $\mathrm{Tb} \mathrm{V}-27$ & $100 \% *$ & $80 \%^{*}$ & $83 \%^{*}$ & $20 \%$ & $50 \%{ }^{*}$ \\
\hline $\mathrm{Tb} \mathrm{V}-28$ & $100 \%{ }^{*}$ & $80 \%{ }^{*}$ & $100 \%{ }^{*}$ & $100 \%{ }^{*}$ & $25 \%$ \\
\hline
\end{tabular}

${ }^{*}$ Significantly different from control group treated with Ringer solution; $P<0.05$, Fisher's exact test.

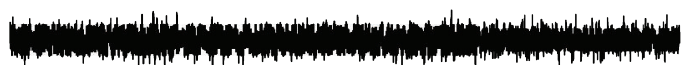

(a)

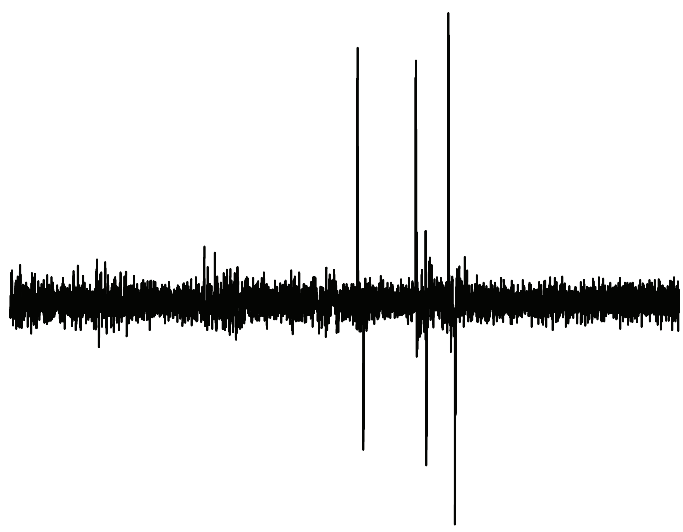

(c)

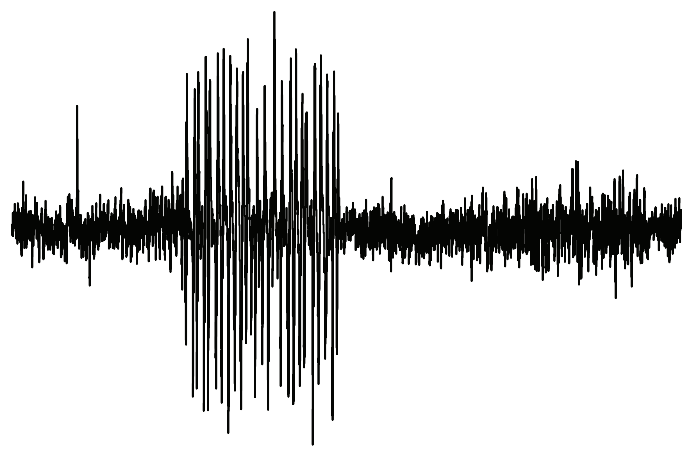

(e)

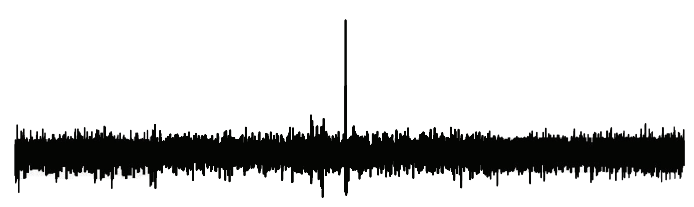

(b)

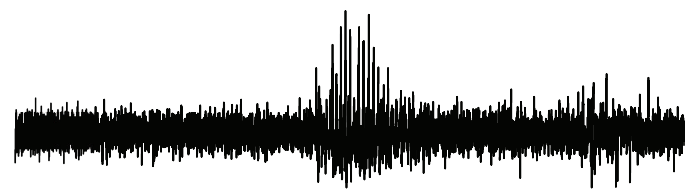

(d)

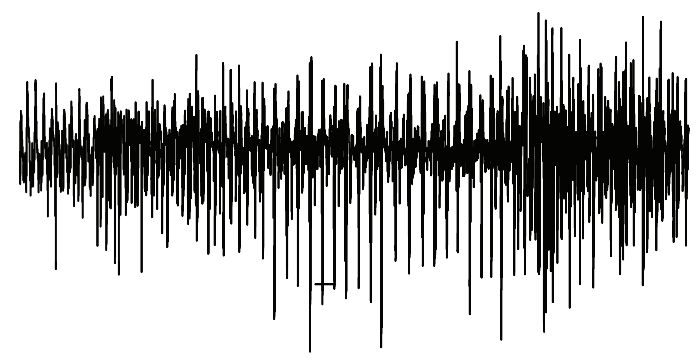

(f)

FIGURE 2: Electroencephalographic recordings to illustrate the alterations observed after injection of toxins into the dorsal hippocampus. (a) Control recording; (b) isolated spikes; (c) grouped spikes; (d) short discharge, (e) moderate discharge; (f) strong discharge.

A large number of studies of toxins obtained from $T$. serrulatus scorpion venom are available. On the other hand, few examine the mechanism of action of toxins purified from T. bahiensis scorpion venom $[4,5]$.

Due to the behavioral and electrographic similarities with toxins from T. serrulatus, the mechanism of action of the toxins utilized in the present study was postulated to involve the enhanced release of neurotransmitters, mainly glutamate, although it has not yet been elucidated.

The more evident behavioral alterations caused by the toxins analyzed in this study were WDS, orofacial automatisms, yawning, and myoclonus, all of them related to abnormal release of neurotransmitters such as glutamate, dopamine, and serotonin. WDS, characterized by shaking 

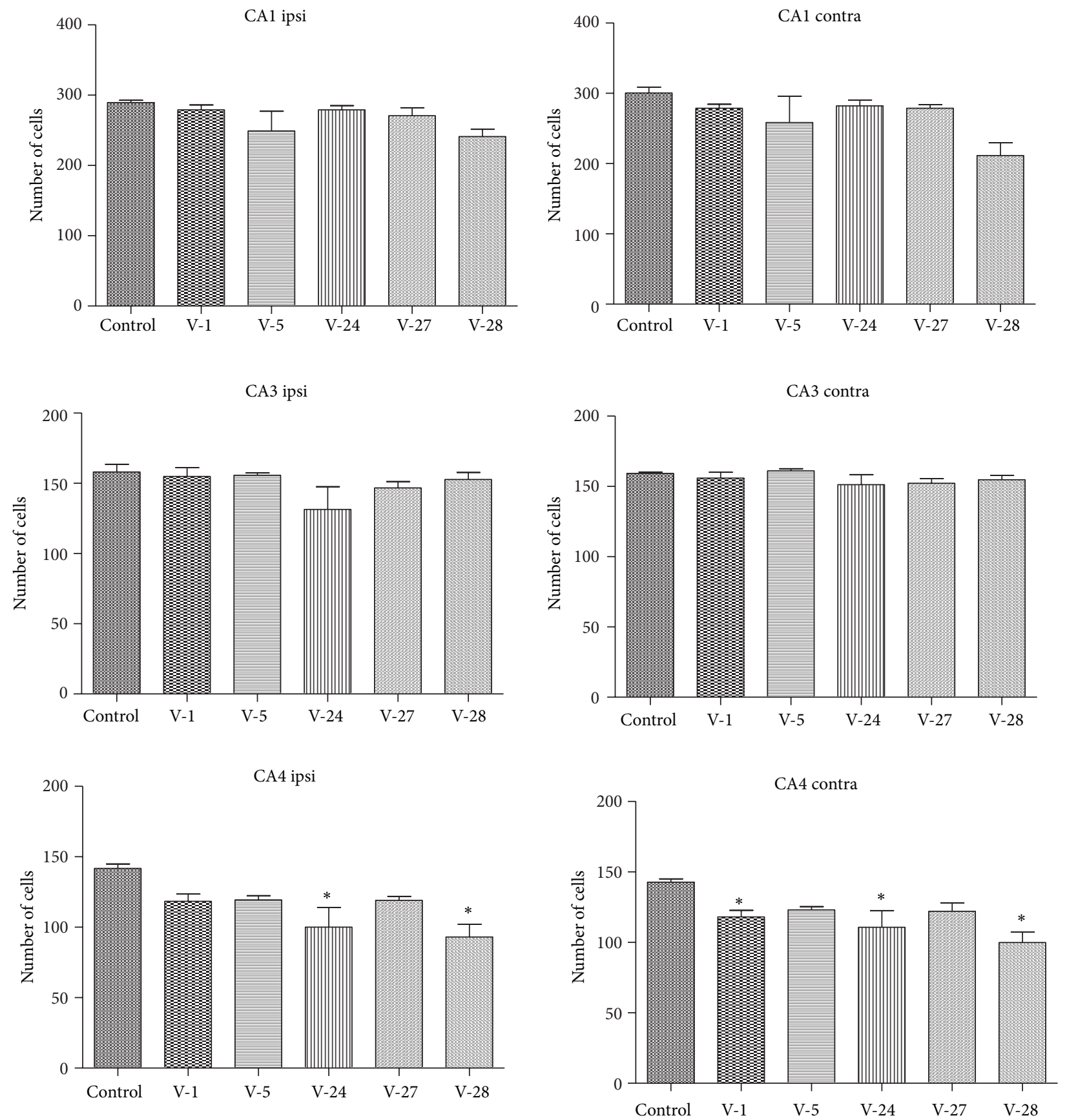

FIGURE 3: Histological analysis of CA1, CA3, and CA4 hippocampal areas after injection of toxins Tb V-1, V-5, V-24, V-27, and V-28 isolated from Tityus bahiensis scorpion venom. "Ipsi" means the side of the injection and "contra" means the opposite area. Data are reported as mean \pm SD of pyramidal neurons. Statistically significant changes within the control and experimental groups were evaluated by ANOVA followed by Tukey's test. $*$ denotes $P<0.05$.

of the head and trunk, is observed in different models of partial epilepsy, such as limbic kindling stimulation or the intralimbic injection of kainic acid or quisqualic acid [22]. The conclusion derived from these studies is that WDS behavior correlates with partial limbic seizures and the number of shakes can be used as an index of the involvement of the limbic structure, in particular, the hippocampus in evoked seizure. Many studies suggest that WDS in rats is mediated by 5 -HT2 receptors $[23,24]$. Orofacial automatisms indicate a stereotyped behavior without apparent function, which can be evoked in rats by apomorphine $[25,26]$ and is restricted to the dopaminergic system [25]. Yawning, in spite of its occurrence in relation to epileptic seizures, which has been rarely described [27], is a common physiological event that occurs with a low frequency in humans and animals, concomitantly with an increase in cortical electroencephalographic activity. 

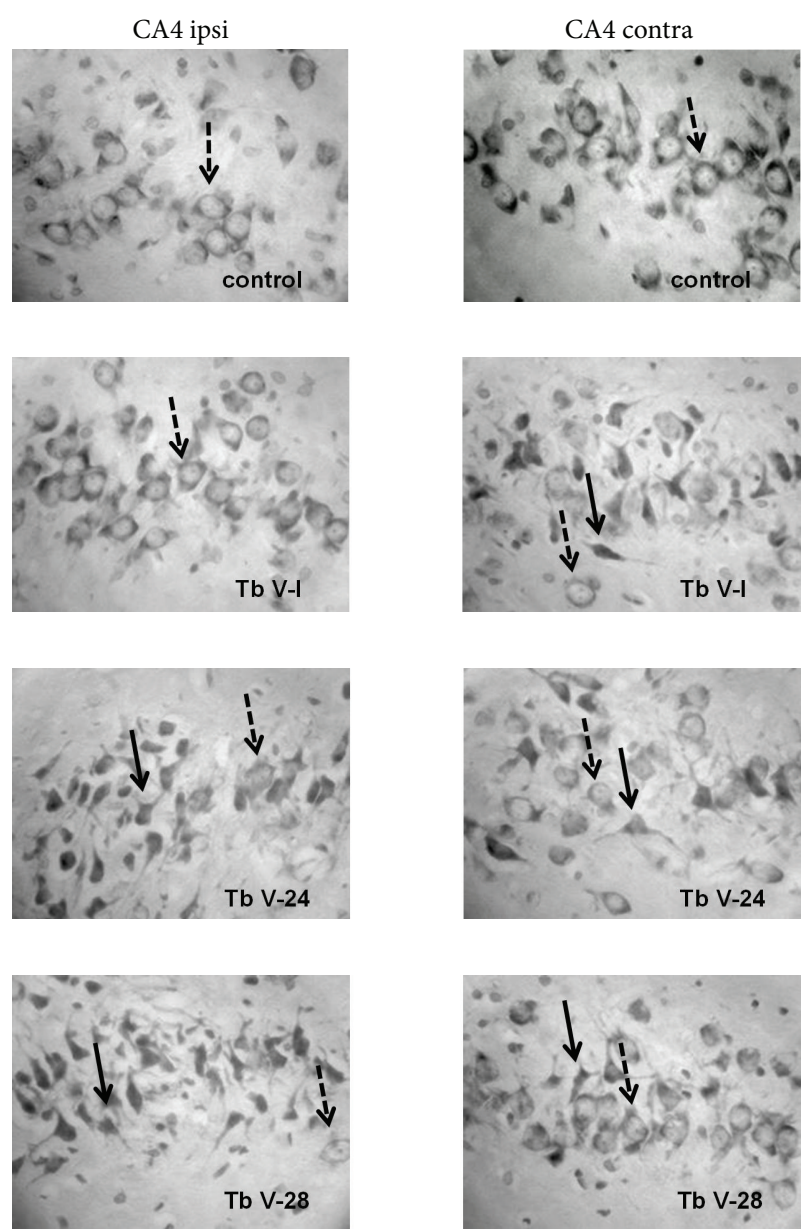

FIgURE 4: Photomicrographs of the CA4 hippocampal area showing a typical pattern of neurodegeneration 7 days after microinjection. The control hippocampus appears to be unaffected. The dashed arrows indicate the intact pyramidal cells, with visible nucleolus. The smooth arrows indicate picnotic neurons. Cresyl violet stain. Magnification: 140x.

While under the control of several neurotransmitters, yawning is largely affected by dopamine [28]. Abnormal repetitive yawning may be the consequence of various local lesions in the central nervous system [29]. Myoclonus presents as a sudden brief jerk caused by involuntary muscle. Epileptic myoclonus refers to the presence of myoclonus in the setting of epilepsy, a chronic seizure disorder [30]. The specific nature of the neuronal circuit abnormality responsible for the generation of myoclonus remains a mystery in any instance [30].

It is interesting to notice that the behavioral alterations observed are more intense when animals were treated with toxins V-1 and V-5 and that the most prevalent electrographic findings in these groups were isolated or grouped spikes and short discharges. On the contrary, the other toxins tested showed lower behavioral signs and the electrographic prevalence was of short and moderate or even intense discharges.

The hippocampus is prone to the generation of epileptiform activity and seizures. There are multiple factors contributing to the epileptogenicity of this structure, including the presence of massive recurrent excitatory connections, the ability of synaptic connections to be strengthened or weakened with repetitive activation, the presence of intrinsically burst-generating cells, and finally a strong influence from ion regulation [16].

Spikes indicate a local disruption of normal function and, as such, are useful in the diagnosis and localization of the underlying pathology generating epileptic seizures. Generalized epileptiform activity can also appear after the initial spike, as well as full tonic or tonic-clonic seizures. The transition from spikes to a full seizure is associated with the maintained or tonic depolarization of the membrane potential and repetitive generation of action potentials [16].

It is possible that the toxins activate different pathways promoting the preferential release of one neurotransmitter or another, thereby causing different behavioral and electrographic effects to appear.

It is well known that scorpion toxins act on ion channels causing changes in their structure and function. Some studies have shown that TsTX toxin, isolated from T. serrulatus scorpion venom, causes an excessive release of glutamate in vitro [20] and in vivo [21] and that TsTX-I, isolated from the same venom, causes a concentration- and time-dependent increase in the release of norepinephrine and epinephrine from bovine adrenal medullary chromaffin cells [31] and produces a selective release of purines from postganglionic sympathetic nerves in the rat vas deferens without modifying the overflow of noradrenaline [32].

All these results seem to indicate that the toxins analyzed here cause alterations in neurotransmitter release. However, more specific studies are necessary to corroborate this notion.

The electrographic effects obtained in the present study are in accordance with data in the literature demonstrating that the injection of scorpion venom or its toxins produces changes in electrographic recordings in rats $[6,21,33,34]$, linking these effects to neurotransmitter release.

Several mechanisms have been implicated in the origin of seizures, including decreased potency of GABAergic inhibition, summation of excitatory synapses, and activation of voltage-sensitive channels [35], and these lead to the triggering of a limited population of neurons. The relationship between excitation and inhibition is important because epileptic activity seems to arise from changes in the balance between them, and any disorder that fosters excitement can lead to excessive and synchronized neuronal activity similar to human epileptiform discharges [35]. Probably the mechanisms involved in this imbalance are inhibitory GABAergic and excitatory glutamatergic transmissions [36].

Various toxins from the venom of Tityus serrulatus and other species such as Tityus bahiensis act on sodium and potassium channels [3] and induce the release of acetylcholine, glutamate and aspartate, and other neurotransmitters in the central and peripheral nervous systems $[3,14,20$, $21,32]$. There are many indications that changes in cholinergic function elicit epileptiform activity. Biochemical studies with pilocarpine, a muscarinic agonist, have shown induction of epileptic activity similar to temporal lobe epilepsy in humans [37]. Glutamate receptor agonists cause epileptiform discharges and neuronal damage in the hippocampus [38]. 
These effects may explain the epileptogenic electroencephalographic changes after scorpion envenomation, because hyperactivation of sodium channels may represent an intrinsic cellular mechanism explaining epileptogenesis [39].

Meanwhile, it is possible that other factors contribute to the occurrence of these epileptic-like effects. The release of cytokines, commonly observed in scorpion envenoming, may have an important role in epileptic-like effects. Several works have demonstrated that scorpion venom and its toxins increase plasma levels of IL- $1 \alpha$, IL- $1 \beta$, IL-6, INF- $\gamma$, and TNF $\alpha$ in moderate or acute accidents [40-42]. Evidence is accumulating that proinflammatory cytokines commonly modify ion channels of virtually every type and that these effects contribute to changes in neuronal excitability [43]. Increasing evidence supports the idea that cytokines are not only inflammatory mediators but also have to be regarded as neuromodulators [43]. It is very important to note that cytokines are able to affect convulsion intensively in rodents, particularly IL- $1 \beta$, which has a proconvulsant activity in a variety of seizure models [44].

Prolonged seizures are associated with neuronal loss due to excitotoxicity in limbic structures [45], mainly the amygdala and hippocampal formation [46]. Our results demonstrated neuronal damage, evidenced by a decrease in the number of cells, in the CA4 area after injection of the toxins Tb V-I, V-24, and V-28. Pyramidal neurons from CA3 and CA4 emit axons that connect with neurons in the CA1 area [47]. Nevertheless, there is no evidence indicating that the contrary is true, and thus we can suggest the existence of a direct or indirect way of stimulation between CA1 (injection site) and CA4. The injection of toxins is not able to reach the contralateral area and cause neuronal loss, but it is possible that the association between neurons in the two areas is responsible for the degeneration. Witter and Amaral [47] asseverate that both sides of the hippocampal formation are connected by means of extensive commissural projections. A hypothesis for neuronal damage is the bidirectional traffic between neurons and glia that involve chemical signals such as ionic gradients, neurotransmitters, trophic factors, and cytokines between these neurons and glia. Some data suggest that a deregulation of these communication mechanisms may contribute to human hippocampal sclerosis [48, 49].

Neurotoxins from scorpion venoms are extremely useful to understand neurotransmission and convulsive activity. We determined that some toxins from T. bahiensis act on CNS when injected into the hippocampus and cause epileptiform activity and neuronal loss. It has been demonstrated that these toxins are excellent tools for this purpose.

\section{Conflict of Interests}

The authors declare that there is no conflict of interests.

\section{Acknowledgments}

This research is part of the Master's thesis presented by Luciene Toshie Takeishi Ossanai to the Post-Graduation Program in Laboratory Research in Public Health of the Disease
Control Coordinating Agency of São Paulo State Public Health Ministry. Thanks are due to the Venom Commission of the Butantan Institute for providing the venom. This work was supported by Fundação de Amparo à Pesquisa do Estado de São Paulo (FAPESP Grant 03/13502-2), CNPq (INCTTox), and Fundação Butantan. Dr. A. Leyva provided the English editing of the paper.

\section{References}

[1] L. Prendini and W. C. Wheeler, "Scorpion higher phylogeny and classification, taxonomic anarchy, and standards for peer review in online publishing," Cladistics, vol. 21, no. 5, pp. 446494, 2005.

[2] FUNASA, Manual de Diagnostico e Tratamento de Acidentes por Animais Peçonhentos, Fundação Nacional de Saúde, Brasília, Brazil, 2nd edition, 2001.

[3] C. T. Cologna, S. Marcussi, J. R. Giglio, A. M. Soares, and E. C. Arantes, "Tityus serrulatus scorpion venom and toxins: an overview," Protein and Peptide Letters, vol. 16, no. 8, pp. 920 932, 2009.

[4] B. Becerril, S. Marangoni, and L. D. Possani, "Toxins and genes isolated from scorpions of the genus Tityus," Toxicon, vol. 35, no. 6, pp. 821-835, 1997.

[5] C. Trequattrini, F. Z. Zamudio, A. Petris, G. Prestipino, L. D. Possani, and F. Franciolini, "Tityus bahiensis toxin IV-5b selectively affects $\mathrm{Na}$ channel inactivation in chick dorsal root ganglion neurons," Comparative Biochemistry and Physiology A, vol. 112, no. 1, pp. 21-28, 1995.

[6] G. A. Lourenço, I. Lebrun, and V. A. C. Dorce, "Neurotoxic effects of fractions isolated from Tityus bahiensis scorpion venom (Perty, 1834)," Toxicon, vol. 40, no. 2, pp. 149-157, 2002.

[7] L. T. T. Ossanai, G. A. Lourenço, A. L. A. Nencioni, I. Lebrun, N. Yamanouye, and V. A. C. Dorce, "Effects of a toxin isolated from Tityus bahiensis scorpion venom on the hippocampus of rats," Life Science, vol. 91, no. 7-8, pp. 230-236, 2012.

[8] M. Ismail, O. H. Osman, K. A. Gumaa, and M. A. Karrar, "Some pharmacological studies with scorpion Pandinus exitialis venom," Toxicon, vol. 12, no. 1, pp. 75-82, 1974.

[9] M. P. Revelo, E. A. Bambirra, A. P. Ferreira, C. R. Diniz, and C. Chávez-Olórtegui, "Body distribution of Tityus serrulatus scorpion venom in mice and effects of scorpion antivenom," Toxicon, vol. 34, no. 10, pp. 1119-1125, 1996.

[10] P. A. Guidine, T. Moraes-Santos, A. R. Massensini, and M. F. Moraes, "Carbamazepine protects the CNS of Wistar rats against the central effects of scorpion envenomation," Neurotoxicology, vol. 29, no. 1, pp. 136-142, 2008.

[11] O. Clot-Faybesse, R. Guieu, H. Rochat, and C. Devaux, “Toxicity during early development of the mouse nervous system of a scorpion neurotoxin active on sodium channels," Life Sciences, vol. 66, no. 3, pp. 185-192, 2000.

[12] E. A. Nunan, M. F. Moraes, V. N. Cardoso, and T. MoraesSantos, "Effect of age on body distribution of Tityustoxin from Tityus serrulatus scorpion venom in rats," Life Sciences, vol. 73, no. 3, pp. 319-325, 2003.

[13] P. A. Guidine, M. B. Mesquita, T. Moraes-Santos, A. R. Massensini, and M. F. Moraes, "Electroencephalographic evidence of brainstem recruitment during scorpion envenomation," Neurotoxicology, vol. 30, no. 1, pp. 90-96, 2009.

[14] A. L. A. Nencioni, G. A. Lourenço, I. Lebrun, J. C. Florio, and V. A. C. Dorce, "Central effects of Tityus serrulatus and 
Tityus bahiensis scorpion venoms after intraperitoneal injection in rats," Neuroscience Letters, vol. 463, no. 3, pp. 234-238, 2009.

[15] G. Paxinos and C. Watson, The Rat Brain in Stereotaxic Coordinates, Academic Press, San Diego, Calif, USA, 4th edition, 1998.

[16] D. A. McCormick and D. Contreras, "On the cellular and network bases of epileptic seizures," Annual Review of Physiology, vol. 63, pp. 815-846, 2001.

[17] R. C. R. de la Vega and L. D. Possani, "Overview of scorpion toxins specific for $\mathrm{Na}^{+}$channels and related peptides: biodiversity, structure-function relationships and evolution," Toxicon, vol. 46, no. 8, pp. 831-844, 2005.

[18] M. A. Romano-Silva, M. V. Gomez, and M. J. Brammer, "Modulation of $\mathrm{Ca}^{2+}$-stimulated glutamate release from synaptosomes by $\mathrm{Na}^{+}$entry through tetrodotoxin-sensitive channels," Biochemical Journal, vol. 304, no. 2, pp. 353-357, 1994.

[19] M. V. Gomez, M. A. Romano-Silva, and M. A. M. Prado, "Effects of tityustoxin on central nervous system," Toxin Reviews, vol. 14, no. 3, pp. 437-456, 1995.

[20] A. R. Massensini, M. A. Romano-Silva, and M. V. Gomez, "Sodium channel toxins and neurotransmitter release," Neurochemical Research, vol. 28, no. 10, pp. 1607-1611, 2003.

[21] A. L. A. Nencioni, I. Lebrun, and V. A. C. Dorce, "A microdialysis study of glutamate concentration in the hippocampus of rats after TsTX toxin injection and blockade of toxin effects by glutamate receptor antagonists," Pharmacology Biochemistry and Behavior, vol. 74, no. 2, pp. 455-463, 2003.

[22] Z. Kleinrok and L. Turski, "Kainic acid-induced wet dog shakes in rats. The relation to central neurotransmitters," NaunynSchmiedeberg's Archives of Pharmacology, vol. 314, no. 1, pp. 3746, 1980.

[23] K. C. Fone, A. J. Robinson, and C. A. Marsden, "Characterization of the 5-HT receptor subtypes involved in the motor behaviours produced by intrathecal administration of 5-HT agonists in rats," British Journal of Pharmacology, vol. 103, no. 2, pp. 1547-1555, 1991.

[24] E. J. Shin, H. J. Jeong, Y. H. Chung et al., "Decrease in the kainate-induced wet dog shake behavior in genetically epilepsyprone rats: possible involvement of an impaired synaptic transmission to the 5-HT2A receptor," Journal of Pharmacological Sciences, vol. 110, no. 3, pp. 401-404, 2009.

[25] B. A. Ellenbroek and A. R. Cools, "Apomorphine susceptibility and animal models for psychopathology: genes and environment," Behavior Genetics, vol. 32, no. 5, pp. 349-361, 2002.

[26] P. J. F. Martins, J. N. Nobrega, S. Tufik, and V. D’Almeida, "Sleep deprivation-induced gnawing-relationship to changes in feeding behavior in rats," Physiology \& Behavior, vol. 93, no. 1-2, pp. 229-234, 2008.

[27] A. E. Yankovsky, F. Andermann, and F. Dubeau, "Post-ictal forceful yawning in a patient with nondominant hemisphere epilepsy," Epileptic Disorders, vol. 8, no. 1, pp. 65-69, 2006.

[28] F. Sanna, S. Succu, M. R. Melis, and A. Argiolas, "Dopamine agonist-induced penile erection and yawning: differential role of D2-like receptor subtypes and correlation with nitric oxide production in the paraventricular nucleus of the hypothalamus of male rats," Behavioural Brain Research, vol. 230, no. 2, pp. 355-364, 2012.

[29] O. Walusinski, "Yawning in diseases," European Neurology, vol. 62, no. 3, pp. 180-187, 2009.

[30] J. N. Caviness, "Myoclonus," Parkinsonism and Related Disorders, vol. 13, no. 3, pp. S375-S384, 2007.
[31] I. M. Conceição, I. Lebrun, M. Cano-Abad et al., "Synergism between toxin- $\gamma$ from Brazilian scorpion Tityus serrulatus and veratridine in chromaffin cells," The American Journal of Physiology-Cell Physiology, vol. 274, no. 6, pp. C1745-C1754, 1998.

[32] I. M. Conceição, A. Jurkiewicz, D. R. Fonseca et al., "Selective release of ATP from sympathetic nerves of rat vas deferens by the toxin TsTX-I from Brazilian scorpion Tityus serrulatus," British Journal of Pharmacology, vol. 144, no. 4, pp. 519-527, 2005.

[33] A. L. A. Nencioni, F. F. Carvalho, I. Lebrun, V. A. C. Dorce, and M. R. L. Sandoval, "Neurotoxic effects of three fractions isolated from Tityus serrulatus scorpion venom," Pharmacology and Toxicology, vol. 86, no. 4, pp. 149-155, 2000.

[34] V. F. Teixeira, I. M. Conceição, I. Lebrun, A. L. A. Nencioni, and V. A. C. Dorce, "Intrahippocampal injection of TsTX-I, a betascorpion toxin, causes alterations in electroencephalographic recording and behavior in rats," Life Sciences, vol. 87, no. 15-16, pp. 501-506, 2010.

[35] E. W. Lothman, E. H. Bertram III, and J. L. Stringer, "Functional anatomy of hippocampal seizures," Progress in Neurobiology, vol. 37, no. 1, pp. 1-82, 1991.

[36] Y. Isomura, Y. Fujiwara-Tsukamoto, and M. Takada, "A network mechanism underlying hippocampal seizure-like synchronous oscillations," Neuroscience Research, vol. 61, no. 3, pp. 227-233, 2008.

[37] G. Zimmerman, M. Njunting, S. Ivens et al., "Acetylcholineinduced seizure-like activity and modified cholinergic gene expression in chronically epileptic rats," European Journal of Neuroscience, vol. 27, no. 5, pp. 965-975, 2008.

[38] G. D. Hilton, J. L. Nunez, L. Bambrick, S. M. Thompson, and M. M. McCarthy, "Glutamate-mediated excitotoxicity in neonatal hippocampal neurons is mediated by mGluR-induced release of $\mathrm{Ca}^{2+}$ from intracellular stores and is prevented by estradiol," European Journal of Neuroscience, vol. 24, no. 11, pp. 3008-3016, 2006.

[39] H. E. Scharfman, "The neurobiology of epilepsy," Current Neurology and Neuroscience Reports, vol. 7, no. 4, pp. 348-354, 2007.

[40] G. D’Suze, S. Moncada, C. González, C. Sevcik, V. Aguilar, and A. Alagón, "Relationship between plasmatic levels of various cytokines, tumour necrosis factor, enzymes, glucose and venom concentration following Tityus scorpion sting," Toxicon, vol. 41, no. 3, pp. 367-375, 2003.

[41] Y. D. Fukuhara, M. L. Reis, R. Dellalibera-Joviliano, F. Q. Cunha, and E. A. Donadi, "Increased plasma levels of IL-1 $\beta$, IL-6, IL-8, IL-10 and TNF- $\alpha$ in patients moderately or severely envenomed by Tityus serrulatus scorpion sting," Toxicon, vol. 41, no. 1, pp. 49-55, 2003.

[42] V. L. Petricevich, "Cytokine and nitric oxide production following severe envenomation," Current Drug Targets: Inflammation and Allergy, vol. 3, no. 3, pp. 325-332, 2004.

[43] M. Schäfers and L. Sorkin, "Effect of cytokines on neuronal excitability," Neuroscience Letters, vol. 437, no. 3, pp. 188-193, 2008.

[44] A. Vezzani, S. Balosso, and T. Ravizza, "The role of cytokines in the pathophysiology of epilepsy," Brain, Behavior, and Immunity, vol. 22, no. 6, pp. 797-803, 2008.

[45] D. G. Fujikawa, S. S. Shinmei, and B. Cai, "Kainic acidinduced seizures produce necrotic, not apoptotic, neurons with internucleosomal DNA cleavage: implications for programmed 
cell death mechanisms," Neuroscience, vol. 98, no. 1, pp. 41-53, 2000.

[46] D. C. McIntyre and K. L. Gilby, "Mapping seizure pathways in the temporal lobe," Epilepsia, vol. 49, no. 3, pp. 23-30, 2008.

[47] M. P. Witter and D. G. Amaral, "Hippocampal formation," in The Rat Nervous System, G. Paxinos, Ed., pp. 635-704, Elsevier, New York, NY, USA, 3rd edition, 2004.

[48] G. Perea and A. Araque, "Communication between astrocytes and neurons: a complex language," Journal of Physiology-Paris, vol. 96, no. 3-4, pp. 199-207, 2002.

[49] M. Tsacopoulos, "Metabolic signaling between neurons and glial cells: a short review," Journal of Physiology-Paris, vol. 96, no. 3-4, pp. 283-288, 2002. 

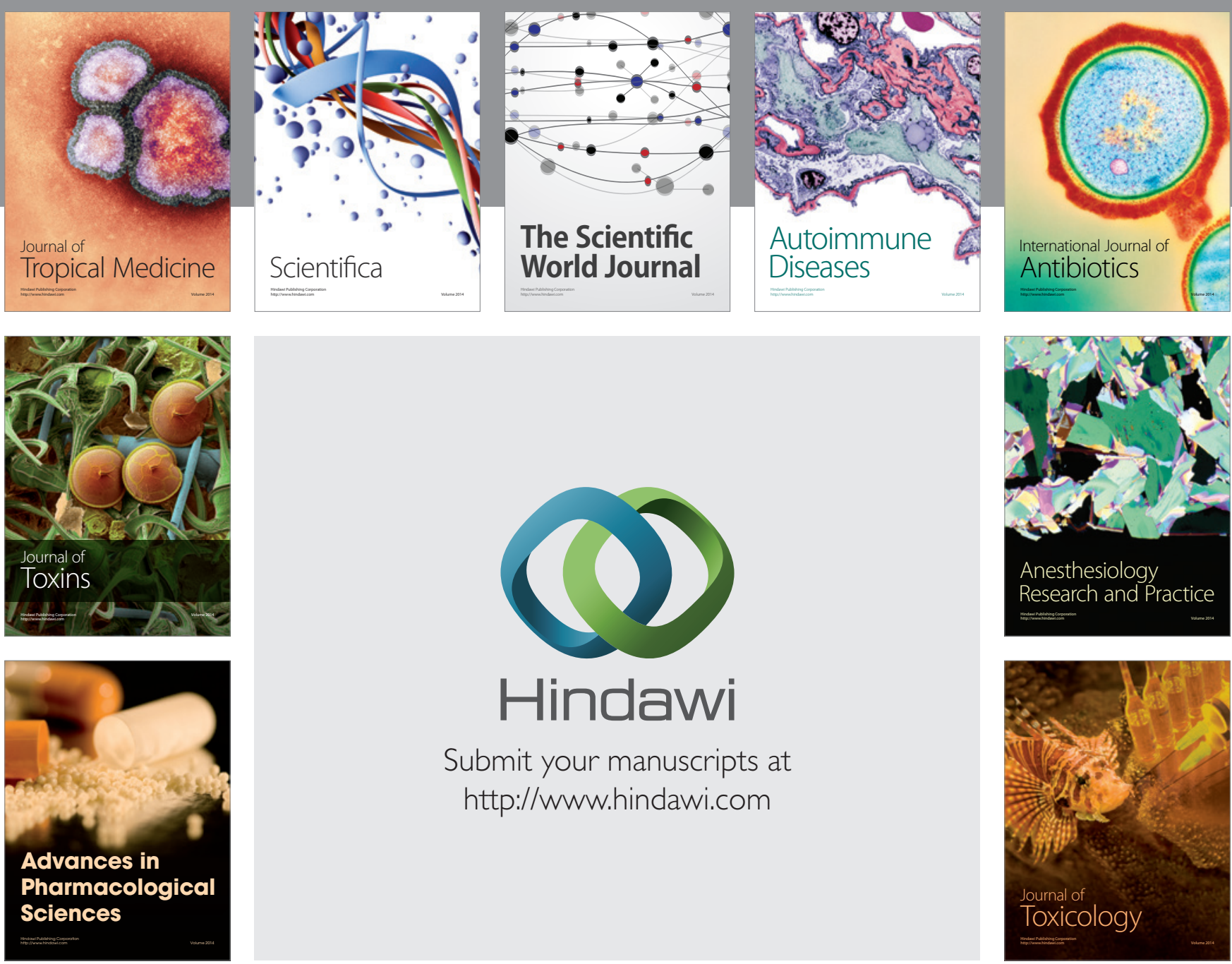

\section{Hindawi}

Submit your manuscripts at

http://www.hindawi.com
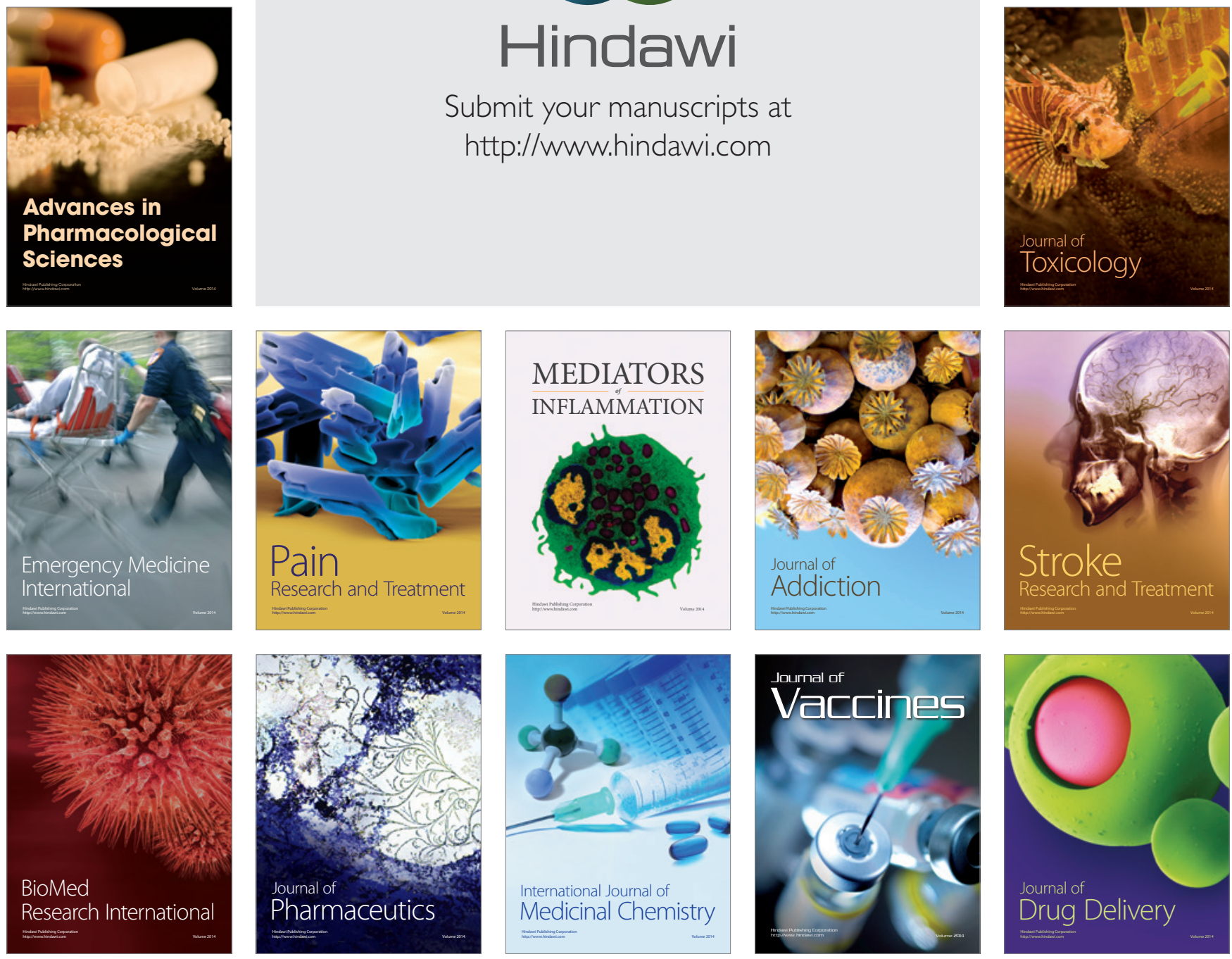